\title{
FEASIBILITY OF RIB KINEMATICS AND INTERCOSTAL-SPACE BIOMECHANICAL CHARACTERIZATION BY ULTRASOUND IN ADOLESCENT IDIOPATHIC SCOLIOSIS
}

\author{
Alisa Hisaund, ${ }^{*}$ Raphä̈l Pietton, ${ }^{\dagger}$ RaphaËl Vialle, ${ }^{\dagger}$ Wafa Skalli, ${ }^{*}$ and Claudio Vergari* \\ * Arts et Métiers Institute of Technology, Université Sorbonne Paris Nord, IBHGC - Institut de Biomécanique Humaine Georges \\ Charpak, HESAM Université, F-75013, Paris, France; and ${ }^{\dagger}$ Department of Pediatric Orthopaedics, Sorbonne Université, Hôpital \\ Armand Trousseau, Assistance Publique-Hôpitaux de Paris (AP-HP), Paris, France
}

\begin{abstract}
The aim of this work was to determine the feasibility of combined ultrasonography and elastography measurement to characterize the mechanical properties of the intercostal space during breathing. Eighteen asymptomatic participants (ages $13 \pm 2$ y) and six participants with adolescent idiopathic scoliosis (AIS) were included $\left(\mathrm{Cobb}\right.$ angle $\left.60^{\circ} \pm 12^{\circ}\right)$. Ultrasonographic and elastographic clips were acquired of $\mathrm{T} 8-\mathrm{T} 9$ ribs and the intercostal space. The two adjacent ribs were tracked to infer the breathing cycle. Shear-wave speed (SWS) was measured in the intercostal space at different stages of the breathing cycle. SWS was symmetric in the control group, during both expiration and inspiration. In AIS, the SWS during inspiration was higher in the convex side than in the concave one $(p=0.02)$. Furthermore, SWS was higher during inspiration than expiration in the control group and in the AIS convex side, but not in the AIS concave side $(p>0.05)$. This new method combining echography and shear-wave elastography allowed measurement of the mechanical characteristics of the intercostal space at different phases of the breathing cycle and highlighted differences between the AIS and control groups. This approach opens the way to further analyses of the biomechanical characteristics of breathing in severe AIS. (E-mail: c.vergari@gmail.com)
\end{abstract}

Key Words: Ultrasonography, Shear wave, Elastography, Scoliosis, Intercostal space.

\section{INTRODUCTION}

Scoliosis is a 3-D deformity of the trunk: it typically presents a lateral shift and axial rotation of the apical vertebra, often together with a flattening of the back. It can result from a neurologic impairment or a congenital malformation of the spine, but the most common form is adolescent idiopathic scoliosis (AIS), the origin of which remains unknown. This specific deformity of the spine can induce a deformity of the rib cage: an asymmetry of the trunk with the appearance of a rib hump and, in more severe cases, an imbalance of the trunk and a respiratory deficiency (Takahashi et al. 2007; Hernandez et al. 2018; Buckland et al. 2020).

Pulmonary function tests are reliable tools to assess respiratory deficiency. However, these tests are quite constraining, and young children are not always

Address correspondence to: Claudio Vergari, Institut de Biomécanique Humaine Georges Charpak, Arts et Métiers ParisTech, 151 boulevard de 1'Hôpital, 75013 Paris, France. E-mail: c.vergari@gmail. com compliant. Moreover, although these tests allow diagnosis of respiratory impairment, they do not always explain the cause underlying the functional limitation. In the case of AIS, respiratory impairment is likely caused by a combination of the rib cage deformity, which can alter the kinematics of the ribs, and the reduced thoracic volume owing to the penetration of the spine in the rib cage. However, it is still not known whether the muscles play a role in reducing respiratory function, or whether they are somehow altered by the deformity. In particular, the intercostal space (IS) is one of the key elements of active respiration, because the thin muscles with alternating pennation directions running between the ribs facilitate the thoracic component of inspiration and expiration (Beyer et al. 2014). Studying the IS seems relevant for a better understanding of pulmonary impairment in scoliosis, especially because intercostal muscle activity appears to be affected in scoliosis (Martínez-Llorens et al. 2010).

Ultrasound elastography is a non-invasive measurement method that has been widely used to measure 
muscle mechanical properties in vivo. It provides a measurement of the local shear-wave speed (SWS) within the tissue, which in turn is related to its elastic modulus (Gennisson et al. 2013). In particular, recent applications have been developed to reliably evaluate the mechanical properties of the soft tissues of the IS (Pietton et al. 2021). However, that study evaluated only the average elastic modulus of the IS during several respiration cycles, whereas studying the variations in elastic modulus at different phases of the cycle could be informative. Furthermore, only elastography was exploited in that study, but simultaneous conventional echography could be used to evaluate the kinematics of the ribs.

The aims of the present study were to determine the feasibility of measuring the IS soft tissue SWS and the kinematics of the ribs during the breathing cycle using ultrasound, and to use this technique to assess asymmetries in people with AIS and alterations relative to an asymptomatic control group. The hypothesis of this study is that there exists a relationship between rib cage geometric asymmetry, muscle shape and function. Hence, we expected that the geometric asymmetry would be reflected in muscle characteristics.

\section{MATERIALS AND METHODS}

\section{Participants}

Data were collected between February 2019 and May 2019 from Trousseau university children's hospital,
Paris, France. Girls were included between the ages of 9 and $17 \mathrm{y}$-an asymptomatic control group $(n=18)$, with no previous musculoskeletal trouble and with scoliosis excluded by clinical examination, and a group with severe AIS $(n=6)$, with Cobb angle $>40^{\circ}$ and main right major thoracic curves. The AIS group was assessed with clinical and radiologic examination. Individuals with concurrent other musculoskeletal trouble or neurological impairment were excluded.

The participants and their parents were informed of the purpose of the study and the functioning of the acquisition, and provided written consent as required by the local ethical committee, which authorized the study.

\section{Data acquisition}

An ultrasonographic exam was performed on each participant using an Aixplorer (version 6.1, Supersonic Imagine, Aix-en-Provence, France) and a linear SL10-2 probe, which allows simultaneous acquisition of conventional B-mode ultrasound and shear-wave elastography (Fig. 1).

An adaptation of a previously described protocol was used (Pietton et al. 2021): first the participant was made aware of the difference between abdominal (diaphragmatic) and thoracic breathing. Then, while lying supine, she was instructed to try to breathe mainly using her rib cage.

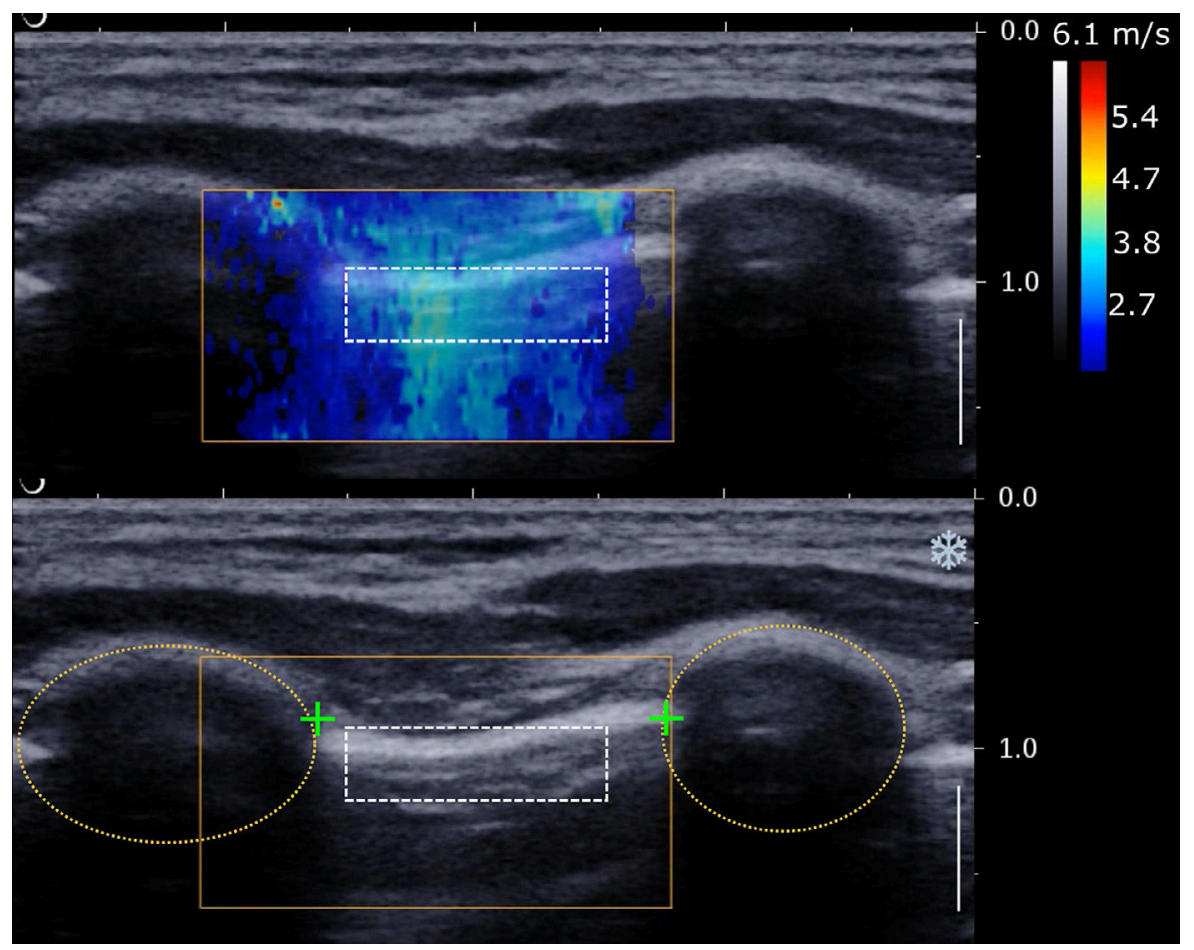

Fig. 1. Example of ultrasound image of intercostal space. Ovals represent the rib cross section, and plus signs the muscle insertions which were tracked during respiration to estimate rib distance. The color chart represents local shear-wave speed. 


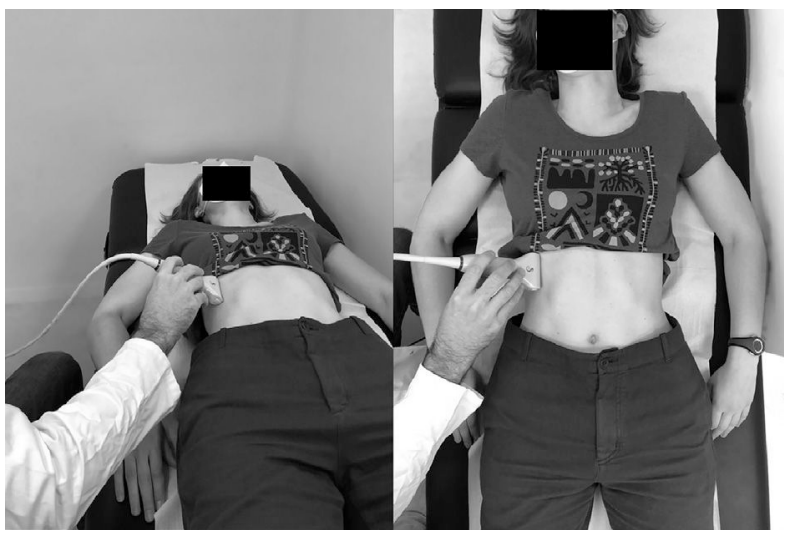

Fig. 2. Example of ultrasound acquisition at $\mathrm{T} 8-\mathrm{T} 9$ intercostal level. The probe is kept orthogonal to the ribs.

Ultrasound measurement was performed at the T8-T9 right and left IS. The probe was placed orthogonally to the ribs and skin, and its anteroposterior position was set at the insertion of the latissimus dorsi and the serratus anterior muscles (Fig. 2). This location was chosen because it is a well-known anatomical ultrasound landmark for serratus plane block (Blanco et al. 2013), and the rib level allows for comfortable measurement while controlling the individual's arm position. Then three clips of $25-40 \mathrm{~s}$ were recorded, taking care to include the two ribs within the field of view during the whole acquisition. The protocol was then repeated on the other side.

During post-processing, a region of interest (ROI) was defined between the ribs to measure the average SWS of the intercostal space. The ROI width was adapted to the observed IS, and its height to the visible layer of soft tissue (about $0.5 \mathrm{~cm}$ ). This region was defined in the first frame of each clip and was semi-automatically tracked in the following frames, to ensure that the same ROI was measured.

All measurements were performed by a single trained operator, but measurements were repeated by a second operator in four participants and on both sides to assess reliability.

\section{Rib kinematics}

The parameter of interest to quantify rib kinematics was the distance between the ribs, which represents how the rib cage expands and retracts locally during respiration. For this purpose, the clips acquired were downsampled at $10 \mathrm{~Hz}$ (from the original $24 \mathrm{~Hz}$ ) and the Bmode echograph (Fig. 1) in each frame was enhanced using a pixel-wise adaptive low-pass Wiener filter and histogram equalization. Since echography and elastography were acquired simultaneously, a light orange rectangle was present in the echographs to represent the elastography measurement region, as shown in Figure 1. This rectangle was static in all images of a given clip, so it was suppressed using an in-painting method consisting of replacing the pixel value with the average intensity value of its surrounding pixels.

The cross sections of the two adjacent ribs were manually annotated in the first echograph (Fig. 1), and then tracked using previously described custom software which allows tracking of an ROI by combining image correlation and minimization of the grayscale difference (Vergari et al. 2016). The 2-D Euclidean minimal distance between the ribs was calculated in each frame, and transformed from pixels to millimeters using the scale available in all clips. The signal of distance during breathing was translated to zero-average, and its zero crossings were used to cut the whole signal into periodic oscillations (Fig. 3). All periods were resampled to the same length of 100 samples to calculate the average ( \pm standard deviation $[\mathrm{SD}]$ ) distance between ribs during normal respiration.

Three parameters of interest were computed: the maximum and minimum distances $\left(D_{\max }\right.$ and $\left.D_{\min }\right)$ and the range of distances $\left(\Delta D=D_{\max }-D_{\min }\right)$. All processing was performed in MATLAB 2018b (The MathWorks, Inc., Natick, MA, USA).

\section{SWS}

The Aixplorer device outputs bitmap movies with color-charted local SWSs (Fig. 1), together with the Bmode images already described.

The clips were processed with a previously described method to extract the SWS of an ROI in a series of frames (Vergari et al. 2014). The ROI was manually defined in the first frame of each clip, and then semi-automatically tracked in the following frames. Then each frame was related to the breathing cycle to estimate the SWS at different instants of the respiration.

Two parameters of interest were computed: the average SWS between $20 \%$ and $40 \%$ of the breathing cycle (inspiration phase, $\mathrm{SWS}_{\max }$ ) and the average between $60 \%$ and $80 \%$ of the breathing cycle (expiration phase, $\left.\mathrm{SWS}_{\mathrm{min}}\right)$.

\section{Statistical analysis and reliability}

In the absence of reference SWS data for this muscle and ROI, we used SWS values obtained in different muscles of the lower and upper limbs (Dubois et al. 2015) to estimate expected differences between groups; with this approach, we determined that a cohort of six people with AIS would be sufficient to detect a difference between full inspiration and expiration with $\alpha<$ 0.05 and power $(1-\beta)>0.9$.

Normality of the data was assessed using the Lilliefors test. The differences between rib cage sides were assessed with either Student's $t$-test, if normality was 


\section{Left side}
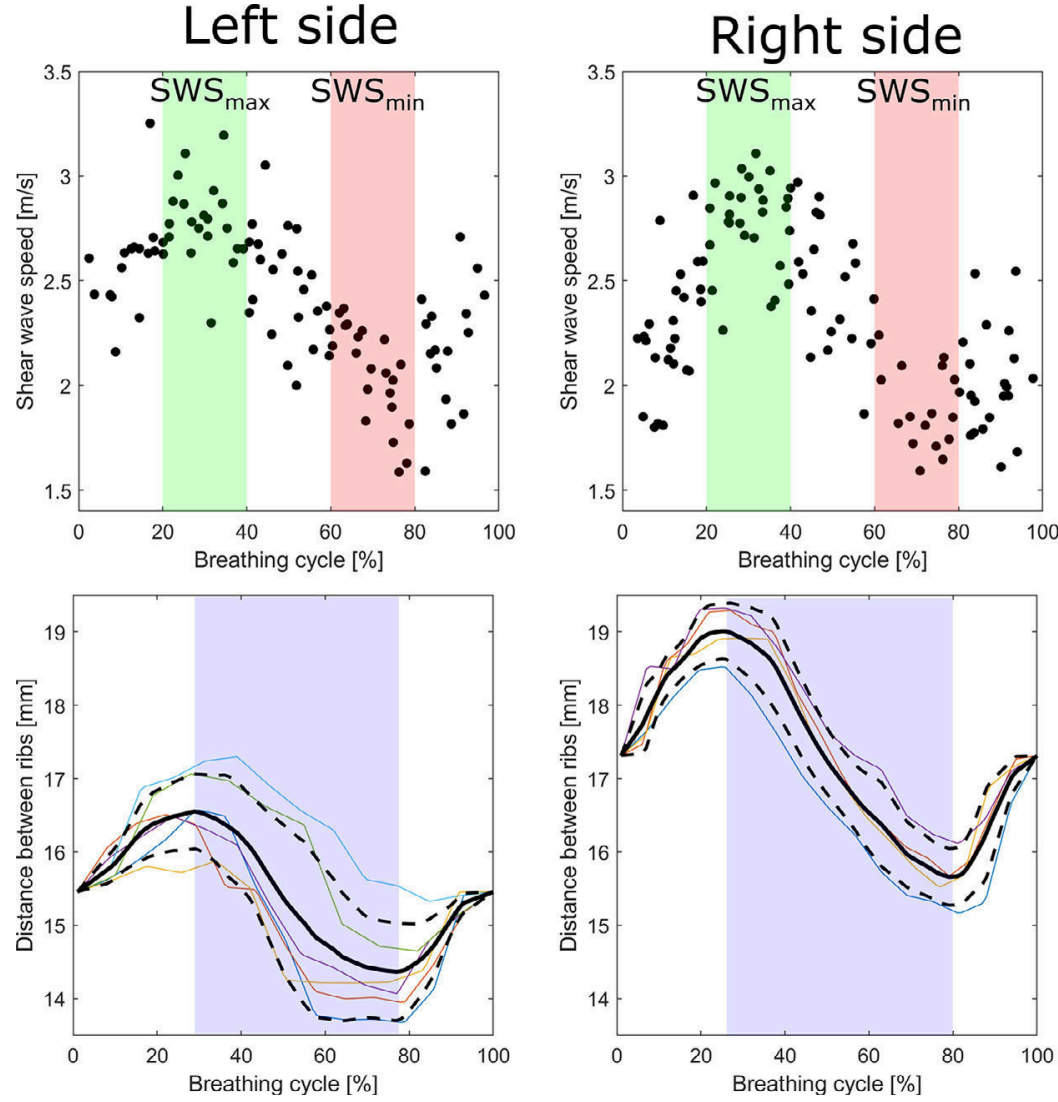

Fig. 3. Example of shear-wave speed (SWS) and inter-rib distance measurement in a participant with scoliosis; the left side is concave and right side is convex. Shaded areas in the SWS graphs represent the regions between $20 \%$ and $40 \%$ (green) and $60 \%$ and $80 \%$ (red) of the breathing cycle, where $\mathrm{SWS}_{\min }$ and $\mathrm{SWS}_{\max }$ were calculated, respectively. In the inter-rib graphs, the colored plots represent several breathing cycles recorded during a single acquisition, while the black solid and dashed lines are their mean $\pm \mathrm{SD}$. The shaded areas in these panels represent a visual guide for the expiration phase (the remainder being inspiration).

proven, or the Mann-Whitney test, if normality was not verified. Paired tests were used when comparing sides or respiration phases, and unpaired tests when comparing groups. Significance was set at $p=0.05$.

The reproducibility of the measurements of inter-rib distance was assessed in the control group in accordance with the ISO 5725 standard and reported as twice the standard deviation of reproducibility; the reproducibility of shear-ware elastography was the subject of a previous study (Pietton et al. 2021).

\section{RESULTS}

Twenty-two asymptomatic participants were included; however, four were excluded because in two cases they could not properly follow instructions concerning normal thoracic respiration. The remaining 18 participants were ages $10-17$ y (average, $13 \pm 2$ y) and had average height $157 \pm 11.4 \mathrm{~cm}$ and weight $46 \pm$ $12 \mathrm{~kg}$. Six participants with AIS were included (ages 16 \pm 2 y), with a Cobb angle between $40^{\circ}$ and $86^{\circ}$ (average, $60^{\circ} \pm 16^{\circ}$ ), average height $167 \pm 3.0 \mathrm{~cm}$ and weight 52 $\pm 1 \mathrm{~kg}$. Differences in height and weight between groups were not significant $(p>0.05)$.

Table 1 shows the reproducibility of the three parameters of interest in the asymptomatic group. Reproducibility was $\leq 3 \mathrm{~mm}$ for each parameter. Figure 3 shows a typical plot of average $( \pm \mathrm{SD})$ distance between the two adjacent ribs during breathing. The positive peak corresponds to maximal rib cage volume in inspiration, the negative peak to the minimal one.

No significant difference was found between the right (convex in AIS) and left (concave) sides in terms of minimum, maximum or range of distance $(p>0.05)$, neither in the asymptomatic nor the AIS group. However, all three parameters were higher in the convex side than the concave side in AIS.

Although results in the AIS group were statistically symmetrical, all distances on the concave side were significantly smaller than in the asymptomatic group ( $p \leq$ 
Table 1. Average, standard deviation and reproducibility (2SD) of the three rib kinematics parameters of interest

\begin{tabular}{|c|c|c|c|c|c|c|c|c|c|}
\hline \multirow[t]{2}{*}{ Parameter } & \multicolumn{3}{|c|}{$D_{\min }(\mathrm{mm})$} & \multicolumn{3}{|c|}{$D_{\max }(\mathrm{mm})$} & \multicolumn{3}{|c|}{$\Delta D(\mathrm{~mm})$} \\
\hline & Control* & $\begin{array}{l}\text { AIS } \\
\text { CX }\end{array}$ & $\mathrm{CV}$ & Control* & $\begin{array}{l}\text { AIS } \\
\text { CX }\end{array}$ & $\mathrm{CV}$ & Control* & $\begin{array}{l}\text { AIS } \\
\text { CX }\end{array}$ & $\mathrm{CF}$ \\
\hline Average & 15.4 & 12.3 & 10.5 & 17.8 & 14.3 & 11.7 & 2.4 & 2.0 & 1.1 \\
\hline SD & 3.5 & 5.1 & 2.4 & 4.0 & 5.8 & 2.6 & 1.0 & 0.9 & 0.5 \\
\hline Reproducibility & 2.7 & - & & 3.0 & - & & 1.0 & - & \\
\hline
\end{tabular}

AIS $=$ adolescent idiopathic scoliosis group $\mathrm{CV}=$ concave side $; \mathrm{CX}=$ convex side $; D=$ distance between ribs

* Left and right sides averaged.

$0.05)$; the difference in minimum distance was at the limit of significance $(p=0.049)$. The convex side, on the other hand, was similar to the asymptomatic group.

\section{SWS}

Values of $\mathrm{SWS}_{\max }$ and $\mathrm{SWS}_{\min }$ showed no significant difference between rib cage sides for the asymptomatic group ( $p>0.05$, Table 2). Therefore, sides were pooled in each participant for subsequent comparisons. Values of $\mathrm{SWS}_{\max }$ were significantly higher than $\operatorname{SWS}_{\min }\left(p=1.9 \times 10^{-5}\right)$; although the average difference was small $(0.1 \mathrm{~m} / \mathrm{s})$, Figure 4 shows that $\mathrm{SWS}_{\max }>$ $\mathrm{SWS}_{\min }$ in all participants.

In the AIS group, $\mathrm{SWS}_{\min }$ was similar in the convex and concave rib cage sides $(p=0.17)$. The $\mathrm{SWS}_{\max }$ of the convex side was significantly higher than for the concave side $(p=0.02)$ and for the asymptomatic group $(p=0.007)$. The SWS ${ }_{\max }$ in the convex side was higher than the concave side in five of six participants; for the sixth, the two sides presented the same value $(2.7 \mathrm{~m} / \mathrm{s})$. The $\mathrm{SWS}_{\max }$ was significantly higher than $\mathrm{SWS}_{\min }$ in the convex side $(p=0.03)$, but not in the concave one $(p=0.7)$.

Pooling $\mathrm{SWS}_{\max }$ and $\mathrm{SWS}_{\min }$, a correlation was observed with inter-rib distance variation (Fig. 5; Spearman's $\rho=0.6, p=0.0008$ ). There was no correlation of SWS with $D_{\min }$ or $D_{\max }$.

\section{DISCUSSION}

The objective of this study was to determine the feasibility of prolonged ultrasound acquisition for observing local rib motion and the mechanical properties

Table 2. Average and SD of the shear-wave speed during inspiration $\left(\mathrm{SWS}_{\max }\right)$ and expiration $\left(\mathrm{SWS}_{\min }\right)$

\begin{tabular}{|c|c|c|c|}
\hline Measurement & $\begin{array}{l}\mathrm{SWS}_{\min } \\
(\mathrm{m} / \mathrm{s})\end{array}$ & $\begin{array}{l}\mathrm{SWS}_{\max } \\
(\mathrm{m} / \mathrm{s})\end{array}$ & $\begin{array}{l}p\left(\mathrm{SWS}_{\min }\right. \\
\left.\text { vs. } \mathrm{SWS}_{\max }\right)\end{array}$ \\
\hline Asymptomatic & $2.1 \pm 0.4$ & $2.2 \pm 0.4$ & $<0.001$ \\
\hline AIS convex side & $2.5 \pm 0.5$ & $2.7 \pm 0.3$ & 0.03 \\
\hline AIS concave side & $2.2 \pm 0.3$ & $2.3 \pm 0.3$ & 0.7 \\
\hline$p$ (asymptomatic vs. convex) & 0.2 & 0.007 & - \\
\hline$p$ (asymptomatic vs. concave) & 0.8 & 0.9 & - \\
\hline$p$ (convex vs. concave) & 0.2 & 0.02 & - \\
\hline
\end{tabular}

AIS $=$ adolescent idiopathic scoliosis.

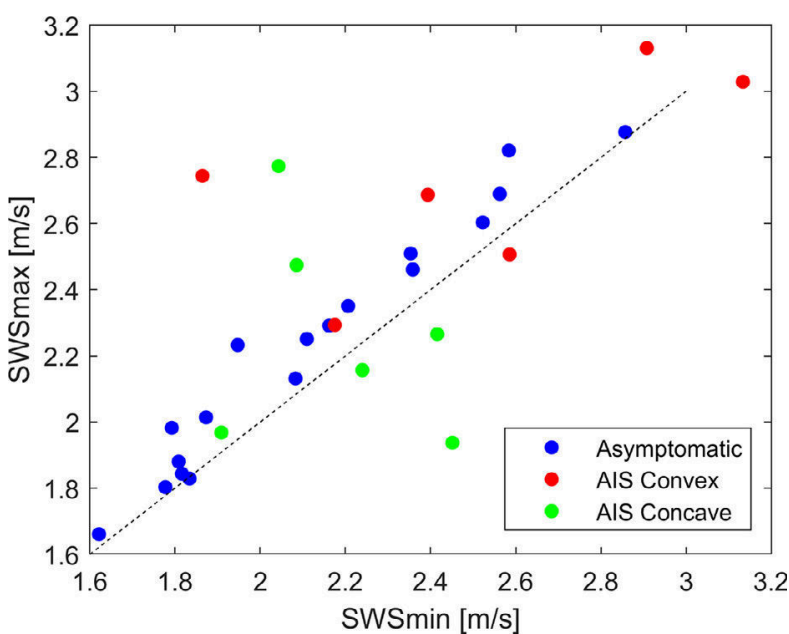

Fig. 4. Maximum and minimum shear-wave speed $\left(\mathrm{SWS}_{\max }\right.$ and $\mathrm{SWS}_{\mathrm{min}}$ ) in the asymptomatic and adolescent idiopathic scoliosis groups. The dashed line is the bisector; all asymptomatic participants are above this line, indicating that $\mathrm{SWS}_{\max }>$ $\mathrm{SWS}_{\text {min }}$, while concave and convex sides of participants with adolescent idiopathic scoliosis can be above or below this line.

of IC soft tissues during the breathing cycle. The proposed protocol allowed estimation of the limits of uncertainty of the method, and provided preliminary results in asymptomatic participants and those with AIS. The proposed approach can give very localized insight into the function of the rib cage during respiration. This is an original and complementary approach to existing methods to study the whole rib cage kinematics or pseudokinematics (Leong et al. 1999; Beyer et al. 2014, 2016; Bastir et al. 2017). Data collection is underway to include a larger cohort with severe AIS as well as mild AIS, which will allow determination of the characteristics of this population more accurately, production of a cartography of the intercostal spaces and potentially definition of novel parameters to describe the elastographic characteristics of the intercostal muscle.

The three parameters chosen to represent the local rib kinematics were symmetric in the asymptomatic group, which was expected, but also in the AIS group. However, the maximum and minimum distance between ribs was significantly higher in the asymptomatic group. 


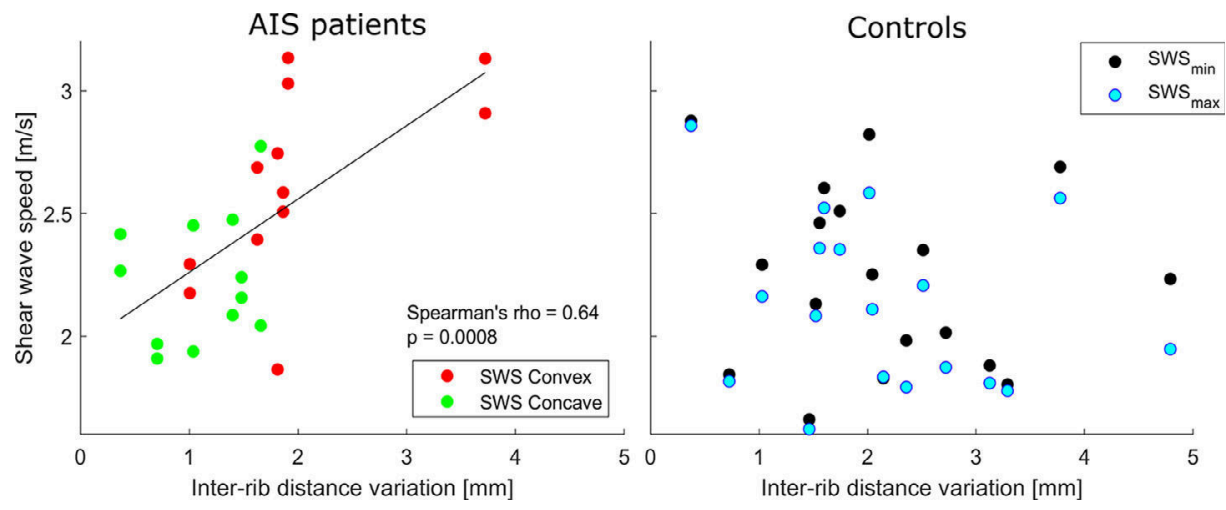

Fig. 5. Relationship between shear-wave speed in the intercostal space and inter-rib distance variation during normal breathing. The relationship was significant in participants with adolescent idiopathic scoliosis $(p=0.0008)$

This corroborates previous research suggesting that individuals with AIS, especially in severe cases, might have reduced lung volume (Chun et al. 2008; Tsiligiannis and Grivas 2012).

Pietton et al. (2021) previously proved the feasibility and reproducibility of SWS measurements of the IS across several breathing cycles; in the present work, the method was extended to assess the variation of SWS all along the breathing cycle. That study obtained an average SWS of $2.1 \pm 0.4 \mathrm{~m} / \mathrm{s}$ and $2.2 \pm 0.3$, respectively, in asymptomatic and AIS groups, with an inter-operator reproducibility of $0.3 \mathrm{~m} / \mathrm{s}$. Our results in the present study were of the same order of magnitude (Table 2), but we observed a higher SWS during inspiration and lower during expiration in both groups. This can be explained by the contraction of the external intercostal muscles which occurs during inspiration, together with passive stretching of both internal and external intercostal muscles while the rib cage expands (Formenti et al. 2020). This stiffening could then be the result of active and passive actions, both phenomena leading to an increase in SWS (De Troyer et al. 2003; Chernak et al. 2013). Conversely, the rib cage muscles relax during expiration, which leads to lower SWS. From a biomechanical point of view, these changes in SWS result from the non-linear mechanical properties of the intercostal muscles and membranes: they stiffen with increased stretching or increasing contraction.

Asymmetries of SWS were detected in the AIS group: SWS at the inspiration phase $\left(\mathrm{SWS}_{\max }\right)$ was higher in the convex than the concave side $(p=0.02)$, and the two respiration phases showed significantly different SWS in the convex side but not in the concave one. This new application of shear-wave elastography opens the way for further research into the functional meaning and clinical relevance of these findings.

Measurements were performed with the participant in a supine position during normal breathing. Pilot data were acquired with an individual in apnea (Pietton et al. 2021), but results were less reproducible than during normal respiration. For this reason, acquisition during deep breathing was not attempted, as it was thought to be even less reproducible. Pilot data were also acquired in standing position, but again, reproducibility was not satisfactory: it was difficult for the operator to maintain a stable and constant probe position during several breathing cycles.

A few limitations of the study can be highlighted. During acquisition, the probe was placed over the ribs to measure the ribs' position and the IS. However, the change of the probe's orientation can alter the perceived distance between ribs, which is only minimized when the probe is orthogonal to both ribs. But it is straightforward to estimate the potential bias: considering an average inter-rib distance of $16.5 \mathrm{~mm}$ and an error in probe orientation of $10^{\circ}$, according to Pythagoras's theorem the measurement error would be $0.25 \mathrm{~mm}$ ( $1.5 \%$ of the global average). To limit this error, care was taken to position the probe so as to minimize the distance between the ribs, and the operator's arm was positioned in a stable position to maintain the probe in the correct orientation during acquisitions.

Indeed, ultrasound examination is not straightforward, and some specific training is necessary for an operator to obtain reproducible results. Furthermore, the subject must be instructed to observe her own rib cage motion and to differentiate thoracic and diaphragmatic breathing. This might not come naturally to everyone, especially in the supine position adopted for this study. Nevertheless, this is an important aspect of the experimental protocol, because almost no rib cage motion can be perceived in diaphragmatic breathing. Hence, participants who cannot or will not follow this protocol (very young children or people with neurological deficits) might not be eligible. Further studies could aim at testing the measurement method in standing or sitting position, when thoracic breathing might come more naturally. 
Finally, SWS quantitative charts are usually shown by the elastographic device with a delay relative to the B-mode images, which in turn appear in real time. This delay has been estimated by Sasaki et al. (2014) to be $1-2 \mathrm{~s}$, which is long enough to affect our measurements. However, results suggest that the delay was shorter in the present study: Figure 3 shows a peak of inter-rib distance between 25\% and $30 \%$ of the breathing cycle, with the peak of SWS only slightly delayed at $30 \%$; this delay was short and not constant in all participants, and therefore it was not corrected. Sasaki et al. used an Aixplorer version 4.2, whereas we used a more recent version (6.1); because the software of this device is continuously updated, it is possible that this delay was shortened in more recent versions. Although not reduced to zero, it was negligible in this study.

\section{CONCLUSION}

Combined echographs and elastography of the IS appears to be a good candidate for investigating the asymmetries of these muscles in people with scoliosis. While the asymptomatic group showed symmetric rib kinematics and intercostal-muscle mechanical properties, participants with scoliosis showed asymmetric muscle properties, with stiffer muscles in the convex side during inspiration. The AIS group also showed smaller movements of the ribs than the asymptomatic group.

While the clinical and biomechanical relevance of these observations must be clarified in future studies, the feasibility of this novel ultrasound application, which is demonstrated in the present work, opens the way for a new line of research.

Acknowledgments - The authors are grateful to the BiomecAM chair program on subject-specific musculoskeletal modeling (with the support of ParisTech and Yves Cotrel Foundations, Société Générale, Covea and Proteor). We are also grateful to Ekaterina Mukhina for her technical help.

Conflict of interest disclosure-The authors have no conflicts to disclose.

\section{REFERENCES}

Bastir M, García-Martínez D, Torres-Tamayo N, Sanchis-Gimeno JA, O’Higgins P, Utrilla C, Torres Sánchez I, García Río F. In vivo 3D analysis of thoracic kinematics: Changes in size and shape during breathing and their implications for respiratory function in recent humans and fossil hominins. Anat Rec John (Hoboken) 2017;300:255-264.

Beyer B, Sholukha V, Dugailly PM, Rooze M, Moiseev F, Feipel V, Van Sint Jan S. In vivo thorax 3D modelling from costovertebral joint complex kinematics. Clin Biomech (Bristol, Avon) 2014;29:434-438.
Beyer B, Van Sint Jan S, Chèze L, Sholukha V, Feipel V. Relationship between costovertebral joint kinematics and lung volume in supine humans. Respir Physiol Neurobiol 2016;232:57-65.

Blanco R, Parras T, McDonnell JG, Prats-Galino A. Serratus plane block: A novel ultrasound-guided thoracic wall nerve block. Anaesthesia 2013;68:1107-1113.

Buckland AJ, Woo D, Vasquez-Montes D, Marks M, Jain A, Samdani A, Betz RR, Errico TJ, Lonner B, Newton PO. The relationship between 3-dimensional spinal alignment, thoracic volume, and pulmonary function in surgical correction of adolescent idiopathic scoliosis: A 5-year follow-up study. Spine (Phila Pa 1976) 2020;45:983-992.

Chernak LA, DeWall RJ, Lee KS, Thelen DG. Length and activation dependent variations in muscle shear wave speed. Physiol Meas 2013;34:713-721.

Chun EM, Suh SW, Modi HN, Kang EY, Hong SJ, Song H-R. The change in ratio of convex and concave lung volume in adolescent idiopathic scoliosis: A 3D CT scan based cross sectional study of effect of severity of curve on convex and concave lung volumes in 99 cases. Eur Spine J 2008;17:224-229.

De Troyer A, Gorman RB, Gandevia SC. Distribution of inspiratory drive to the external intercostal muscles in humans. J Physiol 2003;546:943-954.

Dubois G, Kheireddine W, Vergari C, Bonneau D, Thoreux P, Rouch P, Tanter M, Gennisson J-L, Skalli W. Reliable protocol for shear wave elastography of lower limb muscles at rest and during passive stretching. Ultrasound Med Biol 2015;41:2284-2291.

Formenti P, Umbrello M, Dres M, Chiumello D. Ultrasonographic assessment of parasternal intercostal muscles during mechanical ventilation. Ann Intensive Care 2020;10:120.

Gennisson JL, Deffieux T, Fink M, Tanter M. Ultrasound elastography: Principles and techniques. Diagn Interv Imaging 2013;94:487-495.

Hernandez T, Thenard T, Vergari C, Robichon L, Skalli W, Vialle R. Coronal trunk imbalance in idiopathic scoliosis: Does gravity line localisation confirm the physical findings?. Orthop Traumatol Surg Res 2018;104:617-622.

Leong JCY, Lu WW, Luk KDK, Karlberg EM. Kinematics of the chest cage and spine during breathing in healthy individuals and in patients with adolescent idiopathic scoliosis. Spine (Phila Pa 1976) 2019;24:1310.

Martínez-Llorens J, Ramírez M, Colomina MJ, Bagó J, Molina A, Cáceres E, Gea J. Muscle dysfunction and exercise limitation in adolescent idiopathic scoliosis. Eur Respir J 2010;36:393-400.

Pietton R, David M, Hisaund A, Langlais T, Skalli W, Vialle R, Vergari C. Biomechanical evaluation of intercostal muscles in healthy children and adolescent idiopathic scoliosis: A preliminary study. Ultrasound Med Biol 2021;47:51-57.

Sasaki K, Toyama S, Ishii N. Length-force characteristics of in vivo human muscle reflected by supersonic shear imaging. J Appl Physiol (1985) 2014;117:153-162.

Takahashi S, Suzuki N, Asazuma T, Kono K, Ono T, Toyama Y. Factors of thoracic cage deformity that affect pulmonary function in adolescent idiopathic thoracic scoliosis. Spine (Phila Pa 1976) 2007;32:106-112.

Tsiligiannis T, Grivas T. Pulmonary function in children with idiopathic scoliosis. Scoliosis 2012;7:7.

Vergari C, Mansfield J, Meakin JR, Winlove PC. Lamellar and fibre bundle mechanics of the annulus fibrosus in bovine intervertebral disc. Acta Biomater 2016;37:14-20.

Vergari C, Rouch P, Dubois G, Bonneau D, Dubousset J, Tanter M, Gennisson JL, Skalli W. Non-invasive biomechanical characterization of intervertebral discs by shear wave ultrasound elastography: A feasibility study. Eur Radiol 2014;24:3210-3216. 